ON

\title{
TRANSVERSE FRACTURES OF THE PATELLA.
}

\author{
$\mathbf{B Y}$ \\ JONATHAN HUTCHINSON, F.R.C.S., \\ SURGEON TO THE LONDON HOSPITAL.
}

Rcceired May 17th.-Read June 22nd, 1869.

For some years fractures of the patella have claimed my especial attention, on account of certain features which seem to me to have been somewhat misunderstood. I have had large opportunities for examining them, and have done my best to follow up my cases until their final results became known. Some errors have, I think, become current owing to the result being recorded at much too early a date, and more especially has this been the case as regards "bony union."

On the other hand we are liable to mistakes if we venture to found opinions on museum specimens, to which no life history is attached. My aim has been to connect the circumstances observed in the early stages of these injuries with the conditions discovered in the patient's after-life, or revealed by post-mortem dissection, and thus to get a complete view of the whole case.

With a view to conciseness I have stated my conclusions in a series of propositions. Some of these only express the 
general creed of surgeons, whilst others more or less differ from it.

Prop. 1.-For the purposes of clinical discussion fractures of the patella may be conveniently divided into those in which separation between an upper and lower fragment is possible, and those in which it cannot occur.

Prop. II.-The cases in which no separation of a distal and proximal fragment is possible, are much fewer in number than the others, with which they have scarcely any feature in common.

In this group we count cases of vertical fracture (probably very rare); those cases of starred fracture from blows in which no mobility is possible, and in which probably the articular surface of the bone is not involved; and the not infrequent cases in which fragments of greater or less size are broken off from the borders of the bone.

In none of these can any important questions of treatment arise, it being clear that the limb should be kept at rest, and probable that in many instances bony union will result.

Prop. IIr.-The cases in which separation between the upper and lower fragments is possible, are common, and occur especially in adults who follow laborious occupations.

Prop. IV.-The line of fracture is usually transverse or nearly so, and often near the middle. If one fragment be larger than the other, it will probably be the upper one. Sometimes the lower one is very small. In a few cases the bone is broken into three or more fragments.

Prop. v. - These transverse fractures are not infrequently caused by muscular contraction alone.

Prop. vi.-In the numerous cases in which direct violence is applied to the bone at the moment of fracture, there is almost always muscular contraction simultaneously present, and it is impossible to say which takes the chief share in the result. 
The patella probably never breaks transversely except when the knee is more or less bent, and the bone balanced as a lever on the condyles of the femur. Sudden and unexpected flexion, or the necessity to prevent such, is more likely to break it than extreme flexion. The position of the fracture may serve roughly to indicate the degree of flexion existing at the time the fracture happened. If the fracture is very low down, probably the knee was but little bent, and thus the greater part of the bone still resting on the condyles. I have met with not a few cases in which, as far as the patient's knowledge went, no violence whatever was received on the bone at the time of fracture. In a majority, however, there is a history of the knee having been struck against something during the fall.

Prop. vir.-If the bone is broken by direct violence at a time when the muscle is not in action, there will be no material separation of the fragments either at the time or subsequently; and the probability is very great that the line of fracture will not be transverse.

Unless the bone be held firmly as a lever by the muscles at the time it receives the blow, there is no reason why the fracture should cross the bone transversely, and the probability is that it would be starred. The fact that most fractures are transverse is therefore an argument in favour of the current belief that muscular action is a main agent in causing them. The fact that direct violence under such circumstances cannot possibly rupture the strong fibrous structures by which the bone is everywhere surrounded, explains the circumstance that no subsequent separation is likely to occur.

Prop. virr.-The existence of a wide separation proves that the muscle was in vigorous contraction at the time the bone was broken, and also that the fibrous structures have been extensively lacerated.

The fibrous structures at the sides of the patella are especially strong and are firmly united with it. To admit 
of wide separation there must necessarily be a considerable laceration into the front of the joint. Such laceration is possible only at the time of the accident.

Prop. Ix.- In all cases of transverse fracture involving the whole thickness of the bone, the knee-joint is necessarily implicated, and it is probable that blood will find its way into the joint, and that synovitis with effusion will follow.

The quantity of blood in the joint is probably often very small, but in severe cases it may be considerable. In one case under my observation it distended the joint, and it was necessary ultimately to evacuate it by incision. The effusion of synovia is an almost constant result, and is, I believe, a very important complication of the case.

Prop. x.- There is no persistent contraction of the quadriceps extensor, or of any part of it, after transverse fracture with separation. When the spasmodic effort which attended the fracture ceases, the muscle usually remains completely and permanently at rest.

My experience has invariably been to find the front of the thigh soft and flabby, the quadriceps being quite at rest. I have scarcely ever known the muscle pass into spasm during examination, but in reference to this remark I must state that it does not apply to the first few hours after the accident, respecting which my knowledge is very limited.

Prop. xr.-The subject of a transverse fracture of the patella is usually quite unable by volition.to put the muscle into action, and there is no reason to believe that the muscle is liable to attacks of accidental spasm.

If you ask a man with a recently broken knee-cap to try to lift his limb from the bed, he will reply helplessly, "I cannot;" if you urge him he will get emplatic and 
declare, "Sir, I couldn't do it if you gave me the world," but all the time his quadriceps will make not the slightest movement.

Prop. xII.-After transverse fracture with separation, the fragments become of course widely parted if the knce be bent. If, however, the knee be kept straight, their relative position will not be in the least altered by lifting the limb from the couch and elevating it at right angles with the trunk.

The degree of separation produced by bending the knee after transverse fracture varies considerably in different cases. In some the upper fragment does not follow downwards at all, owing probably to extensive laceration in the fibrous structures. In others, although the fragments are quite movable laterally, there is scarcely any gaping in flexion. The experiment is not one which it is wise to try often, nor except with the utmost gentleness. It is not justifiable except in the earliest stage.

Prop. xiII.-The bringing down of the upper fragment is not as a rule in the least facilitated by the common practice of elevating the limb.

In an uninjured limb at rest in the straight position the patella can be moved upwards or downwards for about three fourths of an inch, the ligamentum patellæ restricting its movement upwards, and the fascia preventing its further passage downwards, whilst the rectus muscle remains perfectly flaccid and offers no sort of impediment. Exactly the same condition of things is met with after transverse fracture. I have tried the experiment of lifting the limb dozens of times, and with great care, but never found the upper fragment to alter in position in the least. Care must of course be taken to make the movements gently, and not to let the knee bend. For years I have never treated fractures of the patella except in the longitudinal position. 
Prop. xIv.-The causes of difficulty in bringing the upper fragment down are several.

1st.-Effusion into the joint lifting the fragments apart.

2nd. Swelling of the torn soft tissues.

3rd. Contraction of the fibrous structures (chiefly met with when the fragments have been pushed apart by swelling which has lasted for some considerable time).

4th. The difficulty there is in getting well hold of the upper fragment, especially when much swelling is present.

Of these probably by far the most constant and the most efficient cause is effusion into the joint. If you examine a case in which there is little or no effusion, there will be little or no difficulty in bringing the fragments close together. In such cases also it is probable that the laceration is but slight, for the laceration, and the effusion which results, will usually be in relation.

The effect of distension of the joint from within in lifting apart the fragments can easily be imagined, and no doubt effusion into the cellular tissue, between the layers of fascia, \&c., will do something towards making the parts rigid, and thus in preventing approximation.

In proportion to the external swelling is the difficulty of getting external pressure, whether manual or by pads, to act efficiently on the upper fragment. The influence of pressure on the sides of the distended joint in making the fluid bulge upwards into the laceration must never be forgotten.

Prop. xv.-In the majority of cases it is easy to force the fragments close and thus obtain crepitus.

Prop. xvr.-The difficulty of securing them in apposition will be exactly in proportion to the amount and duration of the swelling.

I have found much less difficulty in pressing the fragments together, so as to produce crepitus, than books would 
lead us to expect. If difficulty has occurred, it has been from the swelling.

Prop. xvir.-The muscle scarcely ever offers any impediment to the approximation of the fragments.

I do not recollect a single case in which, after the first day or two, even forcible pressure on the upper fragment excited any degree of muscular contraction.

Prop. xviri.-No proof has yet been afforded that transverse fractures of the patella with considerable separation at the time ever unite by bone.

The only proof which we ought to accept on this point is the section of a specimen concerning which the history is definite. There may be such, but I do not know where to refer to them.

Prop. xIx.-It is quite impossible on the living subject to ascertain whether or not bony union has occurred.

In many of my cases at the time of the patient's discharge from the hospital, the fragments were so firmly bound together that I could not produce the slightest lateral movement. In one or two of such cases $I$ have found, by the subsequent stretching of the uniting medium, that it certainly was not bone. A specimen in the London Hospital museum shows such close union, that without section it would have been impossible to say that it was not bone.

Prop. xx.-Transverse fractures with separation may have several different events.

1st. and best.-Close and permanent union of the fragments by fibrous tissue, so that no movement whatever can be detected, and so that section of the bone is needed to show that it is not true bony union.

2nd.-Union by a short and strong ligament.

3rd.-Union by a long ligamentous band which may stretch indefinitely. 
4th.- Entire absence of any uniting medium between the bones; the fibrous capsule of the joint, bursa, \&c., being the only means of connection.

After six weeks' rest and ordinarily careful treatment by back-splint and strapping, it is, I think, easy to obtain close and firm union, in a fourth or even a third of the cases. In another third the union will be close and strong, but still, on forcible pressure, permitting of slight lateral movement, whilst in the remainder union will be weak and with some persisting separation. In the worst cases the separation ought not at this period to be more than a quarter of an inch.

All statements of results made at the date of the patient's leaving the hospitsl are utterly untrustworthy, for in some of the best cases the uniting medium will afterwards stretch. The real result is not known until the patient has been allowed to walk about and bend his knee. A year's interval, at least, ought to be allowed, and even after this it is possible that the fragments may become much more distant.

The assertion that non-union, or membranous union, is a frequent result is, I think, a fallacy.

In all the cases that $I$ have examined at the expiration of treatment, there has been reason to believe that the fragments were connected by a fairly thick uniting medium. Thus they could not be separated beyond a certain length, nor could much lateral movement be produced.

The specimens so common in our museums of wide separation, and in which the thickened capsule, bursa, \&c., are the only connecting structures, are, I suspect, from cases in which premature flexion had been permitted, and the new material stretched until practically lost.

Prop. xxI.-The chance of the best result is greatest in cases in which but little sweling is present, and the fragments are easily approximated; but it by no means always follows that, in cases in which the fragments easily lie quite close, firm union will result. 
Prop. Xxir.-The causes of weak union, or of non-union, are probably two.

1st. The fact that the fragments are usually at some little distance from each other during the first week or two of treatment.

2nd. That the space between the fragments is occupied by a fluid secretion (synovia).

Synovitis is an almost invariable consequence of transverse fractures of any degree of severity, and its effusion usually lasts ten days or a fortnight. During this period approximation is difficult and in many cases cannot be attempted.

Without attributing any special effect to synovia, as synovia, in preventing union, it can easily be understood that the presence of any fluid between the ends of a fractured bone must tend in a most important manner to prevent the consolidation of new material. It will supply a menstruum in which the lymph-cells may be washed away and the plasma dissolved.

Prop. xxirr.-Amongst the causes which help to make actual bony union so exceedingly infrequent, it is, perhaps, fair to suggest that the patella differs from most other bones in having periosteal fibres on one surface only; and that from the direction in which the fracture-force is always applied, all those fibres must invariably be ruptured.

Prop. xxiv.-The objects to be attended to during the treatment are-

1st. To keep the knee straight.

2nd. To get rid, as quickly as possible, of all effusion from the joint.

3rd. To avoid all pressure on the sides of the joint, since such pressure must inevitably force the synovial fluid to bulge forwards into the laceration, and thus separate the fragments. 
4th. To secure the lower fragment as high, and the upper one as low, as possible.

If the effusion be but little, an attempt may at once be made to secure co-aptation; but if otherwise it is better, I think, to wait a while and to use ice to hasten absorption.

Prop. xxv.- Some unexpected results-hitherto, I believe, not described-frequently occur and in some of the best cases prevent the hopes of the surgeon from being fulfilled. Amongst these it may be mentioned that the quadriceps muscle undergoes a very remarkable wasting during the patient's confinement to bed. This wasting is altogether out of proportion to that of the other muscles of his limb and is almost invariably attended by corresponding loss of power.

The wasting of the extensor muscle is, I believe, an almost constant result. If you measure the two thighs, that of the injured side will be much less in girth than the other, and examination will show that the loss of substance is almost wholly in front, the flexors being still plump and large. The patient will be able very easily to put his flexors in action, whilst he will be almost unable to attempt extension, or will do so in a very feeble manner. In many cases he will be absolutely unable to do so, and attempts to lift the limb will result in his bending the knee a little, and then accomplishing a slight elevation chiefly by means of the sartorius.

It is clear that mere disuse will not account for these results; for the flexors must have been, equally with the extensor, at rest during treatment. The explanation must probably be sought in the same physiological law which disables the patient from calling the muscle into action in the early part of the case.

Prop. xxvi.-In probably more than half of the cases of 
transverse fracture of the patella, the quadriceps remains ever afterwards thin and weak, whilst in a not inconsiderable number, it is completely atrophied.

Even in the best cases, -in which the patient regains good power over the extensor and can lift his leg, or kick vigorously,-it is still usual to find the muscle much less bulky than its fellow. In a large proportion the loss of bulk on the front of the thigh is perceptible both to the eye and the hand. I have met with at least six cases of absolute atrophy, so that the bone was quite bare. Most of these were old cases which had been variously treated, but one was in a patient of my own. I do not believe that details of treatment have anything to do with causing or preventing this curious result.

Prop. xxvir.-In almost all cases of fracture with separation, the structures connected with the upper fragment acquire a degree of rigidity during the treatment, which afterwards prevents the upper fragment from easily passing downwards in flexion of the knee.

The condition referred to is, I suppose, one of atrophic contraction. Hunter thought that when separation of the fragments resulted, the extensor muscle must remain too long for its work, and that the patient had to be educated to unusual exertion of it in order to make it act upon the patella and tibia, but, probably, the reverse is the case, and atrophic shortening is the real result.

Prop. XXVIII.-Thus the real danger as to stretching a ligamentous union, is in letting the patient bend the knee, there being little or no danger in allowing him to use his quadriceps.

The function of the extensor muscle has been greatly diminished, both as regards ability to contract and also to relax. It can do neither well. It can neither allow the patella to move downwards in flexion, nor draw it much upwards in extension. After a time it will improve in both voL. LII. 
respects; but, meanwhile, if flexion have been permitted, the uniting ligament will certainly have been stretched.

Prop. xxIx.-Unless the surgeon has in any case deliberately abandoned all hope of benefit from keeping the ligamentous union short, he ought, on no account, to permit even passive flexion of the knee.

Several authorities, more especially Mr. South (see his edition of Chelius), recommend systematicflexion in the sitting posture at an early period, in order to prevent stiffening of the joint. If my conclusions are correct such exercises are certain to stretch the medium of union, and that often very rapidly. I learned the danger from observing this result.

Prop. xxx.- In any case of weak union the surgeon has his choice,-

1st. To let the patient have motion at the knee with the inevitable result of stretched ligament, or

2nd. To keep him permanently with a straight knee and wearing an apparatus.

It may be doubted whether there is any third possibility.

I apply these propositions to all cases in which the fragments are united by fibrous tissue, but not so firmly but that lateral movement can be produced between them. In all such, if flexion is permitted within six months, the uniting structures will stretch, and I do not know that the liability does not extend much longer.

Prop. xxxr.-In cases of close and firm fibrous union (so-called "bony unions") it will be wise to try flexion cautiously. If the patella moves downwards easily then probably it will be perfectly safe.

If the limb can be bent without giving pain, and the whole patella carried downwards as one bone, then the 
result is equivalent to bony union, and probably no ill results will follow from use.

Prop. xxxir.-After complete atrophy of the quadriceps a person can walk very well, and may follow almost any occupation.

The main risk is that owing to the weakness of the limb another fall may occur, and the other patella be broken.

I have met with patients in this condition who could walk twenty or thirty miles a day, and one who was a vandriver and accustomed, with care, to clamber up and down to his box without material inconvenience.

Prop. xxxirr.-The main question for the surgeon in deciding as to whether to advise a patient with weak union to bend the knee or to keep it straight and supported permanently by an apparatus, is as to which plan is the most likely to prevent falss.

The convenience in walking will be found almost equal.

My impression is, that the use of external support adds materially to the patient's safety, and often to his comfort also. I have met with patients, however, who preferred a thin bandage or an elastic knee-cap to a stronger support.

Prop. xxxiv. - The frequency with which the quadriceps atrophies after transverse fractures, suggests the desirability of using galvanism from the first to maintain its nutrition. If used, the limb should, during the process, be raised to the utmost, and the patient made to sit up in bed.

\section{Recapitulation.}

Thus then, briefly to recapitulate, I have tried to show-

1st. That a very remarkable state of physiological 
inactivity in the damaged muscle results from fracture of the patella.

2nd. That the fact of this inactivity destroys the common explanation of the displacement of the upper fragment.

3rd. That this displacement is usnally due, not to muscular traction from without, but to fluid pressure from within.

4th. That the real cause of weak union is the bulging of fluid between the fragments.

5th. That atrophic shortening of the quadriceps often follows these injuries, and, whilst it greatly disqualifies the muscle for extension, renders flexion very dangerous to the uniting ligament.

6th. That an exceedingly interesting and curious occasional result is, permanent and complete atrophy of the muscle concerned.

Should the conclusions which I have stated be confirmed by future experience, it is probable that they will also be found applicable to some other forms of fracture and of injury to tendons. Indeed, it is in the possibility that they may illustrate a physiological law of wide bearing that their chief interest consists. 